\title{
BMJ Open The REDS score: a new scoring system to risk-stratify emergency department suspected sepsis: a derivation and validation study
}

\author{
Narani Sivayoham, ${ }^{\oplus 1}$ Lesley A Blake, ${ }^{1}$ Shafi E Tharimoopantavida, ${ }^{1}$ \\ Saad Chughtai, ${ }^{1}$ Adil N Hussain, ${ }^{1}$ Maurizio Cecconi, ${ }^{2}$ Andrew Rhodes ${ }^{3}$
}

To cite: Sivayoham N, Blake LA, Tharimoopantavida SE, et al. The REDS score: a new scoring system to riskstratify emergency department suspected sepsis: a derivation and validation study. BMJ Open 2019;9:e030922. doi:10.1136/ bmjopen-2019-030922

\section{- Prepublication history for} this paper is available online. To view these files, please visit the journal online (http://dx.doi. org/10.1136/bmjopen-2019030922).

Received 07 April 2019 Revised 29 July 2019 Accepted 02 August 2019
Check for updates

(C) Author(s) (or their employer(s)) 2019. Re-use permitted under CC BY-NC. No commercial re-use. See rights and permissions. Published by BMJ.

${ }^{1}$ Emergency Department, St Goerge's University Hospitals NHS FT, London, UK

${ }^{2}$ Anaesthesia and Intensive Care Humanitas Research Hospital and Humanitas University, Milan, Italy

${ }^{3}$ Adult Intensive Care Unit, St George's Healthcare NHS Trust and St George's University of London, London, UK

Correspondence to Narani Sivayoham; Narani.Sivayoham@stgeorges. nhs.uk

\section{ABSTRACT}

Objective To derive and validate a new clinical prediction rule to risk-stratify emergency department (ED) patients admitted with suspected sepsis.

Design Retrospective prognostic study of prospectively collected data.

Setting ED.

Participants Patients aged $\geq 18$ years who met two Systemic Inflammatory Response Syndrome criteria or one Red Flag sepsis criteria on arrival, received intravenous antibiotics for a suspected infection and admitted.

Primary outcome measure In-hospital all-cause mortality.

Method The data were divided into derivation and validation cohorts. The simplified-Mortality in Severe Sepsis in the ED score and quick-SOFA scores, refractory hypotension and lactate were collectively termed 'component scores' and cumulatively termed the 'Riskstratification of ED suspected Sepsis (REDS) score'. Each patient in the derivation cohort received a score (0-3) for each component score. The REDS score ranged from 0 to 12 . The component scores were subject to univariate and multivariate logistic regression analyses. The receiver operator characteristic (ROC) curves for the REDS and the components scores were constructed and their cut-off points identified. Scores above the cut-off points were deemed high-risk. The area under the ROC (AUROC) curves and sensitivity for mortality of the high-risk category of the REDS score and component scores were compared. The REDS score was internally validated.

Results 2115 patients of whom 282 (13.3\%) died in hospital. Derivation cohort: 1078 patients with 140 deaths (13\%). The AUROC curve with $95 \% \mathrm{Cl}$, cut-off point and sensitivity for mortality $(95 \% \mathrm{Cl})$ of the high-risk category of the REDS score were: derivation: 0.78 (0.75 to 0.80 ); $\geq 3$; 85.0 (78 to 90.5). Validation: 0.74 (0.71 to 0.76 ); $\geq 3$; 84.5 (77.5 to 90.0). The AUROC curve and the sensitivity for mortality of the REDS score was better than that of the component scores. Specificity and mortality rates for REDS scores of $\geq 3, \geq 5$ and $\geq 7$ were $54.8 \%, 88.8 \%$ and $96.9 \%$ and $21.8 \%, 36.0 \%$ and $49.1 \%$, respectively.

Conclusion The REDS score is a simple and objective score to risk-stratify ED patients with suspected sepsis.
Strengths and limitations of this study

- The broad inclusion criteria allowed the inclusion of a wide variety of patients with suspected sepsis.

- Patients with missing data were not excluded.

- Patients whose final diagnosis was not related to an infection/sepsis were included.

- Patients discharged from the emergency department were not studied.

- A single-centre study that requires external validation.

\section{INTRODUCTION}

Sepsis, a life-threatening condition, ${ }^{1}$ is best managed by early recognition and treatment. ${ }^{2}$ The majority of patients in hospital with sepsis are admitted as an emergency with community acquired sepsis. ${ }^{3}$ It is therefore important to identify patients at highest risk of death in the emergency department (ED). Identification of the high-risk patients will both facilitate increased focus of care and also helps determine the transfer destination. In addition, it facilitates the initiation of a conversation on prognosis.

Patients at high-risk of death may be identified by using an evidence based risk-stratification score. The ED is a pressured environment and any score that is used should be easy to calculate using readily available variables. The objective criteria defining sepsis, a minimum two-point increase, ${ }^{1}$ in the Sequential Organ Failure Assessment (SOFA) score, ${ }^{4}$ corresponds to a mortality rate of over $10 \%$. Calculation of the change in SOFA score in a busy $\mathrm{ED}$ is at best labour intensive, as it requires the calculation of the presenting and baseline SOFA scores, and at worst inaccurate as some of the criteria are not met or routinely measured in the ED.

The quick-SOFA (qSOFA) score $^{5}$ was created to risk-stratify patients without the 
need for blood tests and has three variables: respiratory rate $(\mathrm{RR}) \geq 22 / \mathrm{min}$, systolic blood pressure $(\mathrm{SBP}) \leq 100$ $\mathrm{mm} \mathrm{Hg}$ and altered mental state. The presence of $\geq 2$ variables places the patient in a high-risk category for mortality. Prospective validation studies of the qSOFA score in the ED have found the sensitivity for mortality to vary between $30 \%$ and $70 \%,{ }^{6-9}$ a rule-in score that cannot be used alone. The Mortality in Severe Sepsis in the ED score (MISSED score) ${ }^{1011}$ is another scoring system that has been derived, validated and simplified. The simplified-MISSED (sMISSED) score $^{12}$ has been externally validated, ${ }^{13}$ and like the qSOFA score has only three variables: age $\geq 65$ years, serum albumin $\leq 27 \mathrm{~g} / \mathrm{L}$ and an international normalised ratio (INR) $\geq 1.3$, scoring one point each when present; scores of $\geq 2$ places the patient in a high-risk category for mortality.

The most widely studied high-risk criteria in sepsis are refractory hypotension (RH); ( $\mathrm{RH}=$ low blood pressure (BP) after an adequate fluid bolus needing vasopressors to maintain a mean arterial pressure (MAP) $>65 \mathrm{~mm}$ $\mathrm{Hg}),{ }^{1}$ and high lactate (HL); HL=lactate $\geq 4 \mathrm{mmol} / \mathrm{L} .{ }^{14}{ }^{15}$ But not all patients who go on to die meet these criteria while in the ED. We have previously reported that $71 \%$ of patients who die in hospital can be identified by using a combination of a sMISSED score of $\geq 2$, RH and HL. ${ }^{12}$

The aim of this study is to derive and validate a new scoring system combining the SMISSED and qSOFA scores with RH and lactate, to Risk-stratify ED suspected Sepsis (REDS) patients for in-hospital mortality. The cut-off point of the receiver operator characteristic (ROC) curve will stratify patients into high-risk and low-risk categories. The performance of the REDS score will be compared with that of the sMISSED and qSOFA scores, RH and lactate based on the following criteria:

1. the area under the ROC (AUROC) curve and

2. the sensitivity for mortality in the high-risk category.

\section{METHODS}

\section{Study design, time period and setting}

A prospectively collected convenience sample of adults aged $\geq 18$ years who received intravenous antibiotics in the ED for suspected sepsis and admitted between 8 February 2016 and 31 August 2017 were studied. The study population was divided chronologically in half into derivation and validation cohorts, by dividing the population along the number of deaths. This study was carried out in the ED of an urban University Teaching major trauma centre with 120000 annual adult attendances.

\section{Participant selection and measurements}

Inclusion criteria: all patients presenting to the ED who met the criteria in either of the following two categories:

1. Patients who met any one of the following Red Flag ${ }^{16}$ criteria on arrival:

- Unwell within 6 weeks of chemotherapy.

- $\mathrm{RR}>25 / \mathrm{min}$.

- Heart rate $(\mathrm{HR})>130 / \mathrm{min}$.
- $\mathrm{SBP}<90 \mathrm{~mm} \mathrm{Hg}$ or MAP $<65 \mathrm{~mm} \mathrm{Hg}$.

- Reduced alertness.

- New requirement for oxygen to maintain saturations $>90 \%$.

- Temperature $\leq 35^{\circ} \mathrm{C}$.

- A non-blanching rash.

2. Patients who met any two of the following expanded Systemic Inflammatory Response Syndrome (SIRS) ${ }^{17}$ criteria on arrival:

- $\mathrm{RR}>20 / \mathrm{min}$.

- $\mathrm{HR}>90 / \mathrm{min}$.

- Temperature $<36^{\circ} \mathrm{C}$ or $>38^{\circ} \mathrm{C}$.

- White cell count $<4$ or $>12 \times 10^{9} / 1$ (if available on arrival).

- New confusion.

- Blood glucose $>7.7 \mathrm{mmol} / \mathrm{L}$ in non-diabetics.

Data were collected retrospectively from the ED notes and the electronic patient records and entered into an electronic spreadsheet. All data were checked by a second researcher for accuracy. The data collected were as follows: date of admission, method of arrival, initial vital signs (RR, HR, BP, oxygen saturations, temperature, blood glucose, altered mental status), final BP, initial lactate, serum albumin, INR, the use of warfarin or directly acting oral anticoagulants, white cell count, in those patients who received a minimum $2 \mathrm{~L}$ of fluids the BP and lactate after the second litre of fluid, the ability to live independently, final diagnosis, ICU admission, date of discharge and outcome at discharge. The highest RR, lowest SBP and highest lactate in the ED was also collected for the derivation cohort. The number of SIRS criteria, ${ }^{17}$ met, do not attempt resuscitation (DNAR) orders made in the community and the ED were noted. Hospital length of stay (HLOS) was calculated from the date of admission and discharge. The following were coded as RH: MAP of $<62 \mathrm{~mm} \mathrm{Hg}$ after a minimum $2 \mathrm{~L}$ fluid bolus or $30 \mathrm{~mL} / \mathrm{kg}$, a MAP $\leq 55 \mathrm{~mm} \mathrm{Hg}$ after less than $2 \mathrm{~L}$ of fluid or commencement of vasopressors in the ED. Patients who had vasopressors commenced only to compensate hypotension induced by anaesthetic agents were not coded as RH.

\section{Outcome measures}

Primary endpoint: n-hospital all-cause mortality. Patients discharged on a palliative care pathway and died within 15 days of discharge were counted as in-hospital deaths.

\section{Data analysis}

The baseline characteristics of the derivation and validation populations were compared to understand if there were any difference in the populations.

\section{Derivation and construction of the REDS score}

The OR for mortality for HL (initial lactate) and RH were calculated and compared with the OR for mortality of the high-risk qSOFA (initial vital signs) and sMISSED scores. $\mathrm{RH}$ and lactate were transformed into scoring systems by the allocation of scores $0-3$. Each patient had a qSOFA, 
sMISSED, RH and a lactate score (collectively referred to as component scores) calculated. The component scores were subject to univariate analysis (UVA) followed by multivariate logistic regression (MVLR) and construction of a MVLR model. The cumulative value of the component scores formed the REDS score. ROC curves were constructed for the REDS score and the component scores for the primary point. The AUROC curves were calculated and compared. The optimal cut-off point as determined by the statistical software programme was used to stratify patients into high-risk and low-risk categories. The test-characteristics of the high-risk category of the REDS score and the component scores were calculated. The REDS score was validated in the latter half of the data set. Mortality rates associated with each score $0-3$ for the component scores and 0-12 for the REDS score were calculated.

MedCalc Statistical Software V.18.1 was used for statistical analysis. UVA on continuous data was tested using the Student's t-test when normally distributed and the Mann-Whitney test when normality was rejected. The $\chi^{2}$ test was used for categorical data. Statistical significance was defined as $\mathrm{p}<0.05$.

An MVLR model was constructed using the 'Enter' method. Variables were entered if $\mathrm{p}<0.05$ and removed if $p>0.1$. The OR for mortality in the MVLR model was assessed for the need to weight the scores. Discrimination and calibration of the model were assessed by the AUROC curve and the Hosmer-Lemeshow test, ${ }^{18}$ respectively. The difference in AUROC curves was assessed by the DeLong method. ${ }^{19}$

\section{Variants of the REDS score in the derivation cohort \\ Alternative qSOFA scores in the REDS score}

The REDS score was recalculated using the final RR and SBP and the highest RR and lowest SBP in the qSOFA score, mental state was assumed to be unchanged as any subtle changes may not have been accurately documented.

\section{The highest lactate in the REDS score}

The REDS score was recalculated using the highest lactate instead of the initial lactate.

\section{$\mathrm{RH}$ as a homogeneous population in the REDS score}

The REDS score was recalculated assuming that RH was a homogeneous population, thereby not stratifying RH by the associated lactate.

\section{Exclusion of patients with missing variables}

The REDS score was also studied after excluding the population of patients with missing variables but maintaining those who were on oral anticoagulants, although scoring 0 for INR.

The AUROC curves, the cut-off points and the test-characteristics of the high-risk group were also studied for these variants of the REDS score.

\section{Validation study}

The REDS score was validated in the latter part of the data set. This was done by calculating the REDS score for each patient, constructing ROC curves, identifying the cut-off point, stratifying patients by the cut-off point and studying the test-characteristics of the high-risk group.

\section{Sample size and missing variables}

Steill and Wells ${ }^{20}$ advocate a minimum 10 outcomes per variable. An adequate sample size for the derivation and validation populations would have a minimum 80 deaths each when values for all eight variables (age, BP, mental status, RR, INR, serum albumin, lactate and RH) were present. Missing variables were assumed to be normal. Those on oral anticoagulants were given a score of 0 for INR. This pragmatic approach would reflect future clinical use.

\section{Patient and public involvement}

No patients or the public were involved in the development of the research question or conduct of the study.

\section{Data availability}

All data used are included in the manuscript.

\section{RESULTS}

Baseline characteristics of the derivation and validation populations are found in table 1 . The baseline characteristics of the derivation and validation populations were similar. The difference in the percentage of patients admitted to the ICU is likely to reflect seasonality. Of the 120 patients with $\mathrm{RH}$, only seven received less than $2 \mathrm{~L}$ of fluid.

\section{Derivation study}

Of the 1093 patients collected between 8 February and 4 October 2016, ED notes were unavailable for 15 . Of the remaining 1078 patients 140 died. Missing results: lactate 54, serum albumin 49, INR 107 and another 107 were on oral anticoagulants. Ninety of the 791 patients with results for all variables and not on oral anticoagulants, died. Normality was rejected for all continuous variables.

The OR for mortality for HL and RH were similar to that for the high-risk categories of the SMISSED and qSOFA scores (table 2A).

Lactate and RH were transformed into scoring systems by allocating scores which had mortality rates similar to the corresponding scores in the sMISSED and qSOFA scores, as described in figure 1 . Lactates $\geq 4 \mathrm{mmol} / \mathrm{L}$ were scored 3. 'Low-risk' lactates $(0-3.9 \mathrm{mmol} / \mathrm{L})$ were further stratified, ${ }^{21}$ : lactates $2.1-3.9 \mathrm{mmol} / \mathrm{L}$ were scored 1 and $\leq 2 \mathrm{mmol} / \mathrm{L}$ scored 0 . The overall mortality rate of $39.7 \%$ for RH would indicate a score of 3 for all RH. RH is known to have a higher mortality rate when associated with a lactate $>2 \mathrm{mmol} / \mathrm{L} .^{22}$ Therefore, $\mathrm{RH}$ was scored 3 when the associated lactate was $>2 \mathrm{mmol} / \mathrm{L}$ after $2 \mathrm{~L}$ of crystalloid and scored 2 if the lactate was $\leq 2 \mathrm{mmol} / \mathrm{L}$. When the lactate postfluid bolus was not available, the initial lactate 


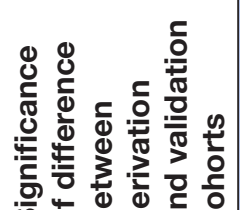

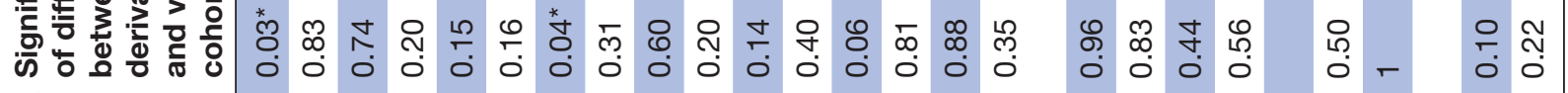

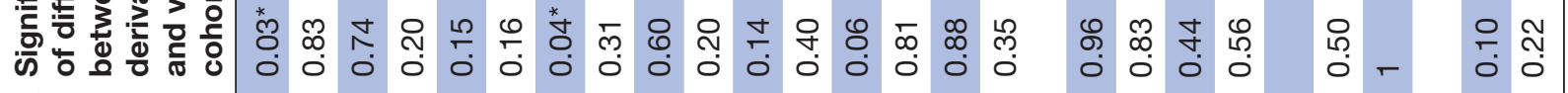

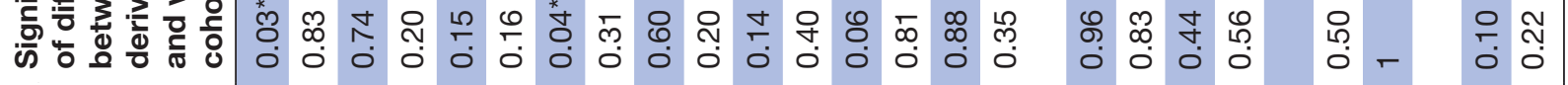

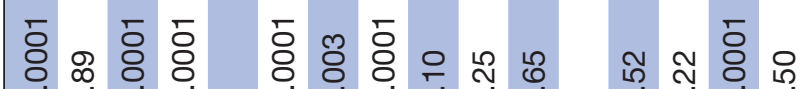

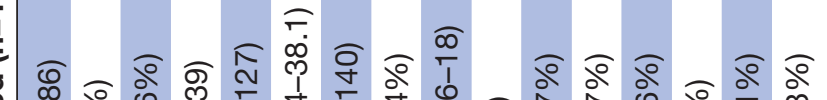

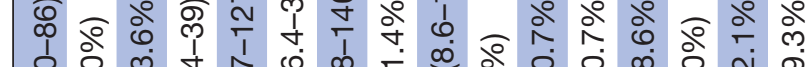

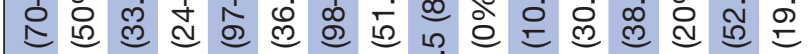
암

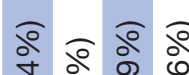

वे वे

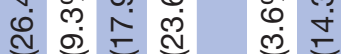

วั

ते $m$ ए

กิ่

咅员

o.

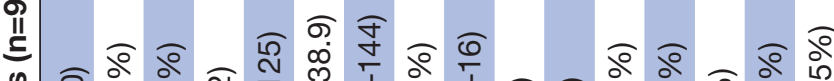

ต व

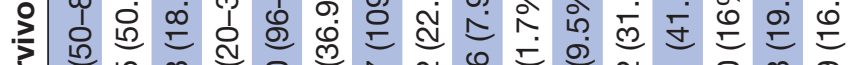

ゆ)

a

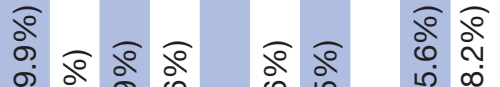

एं

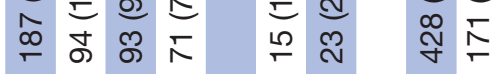

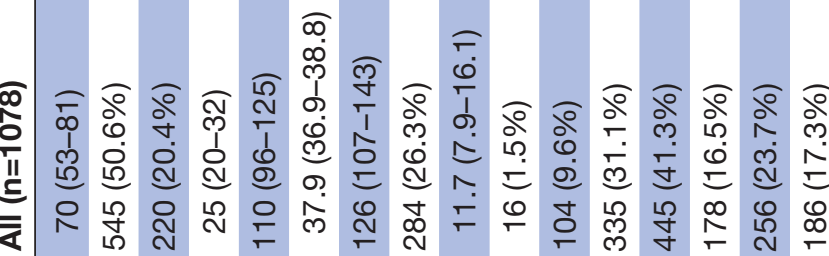

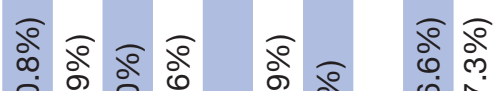

空

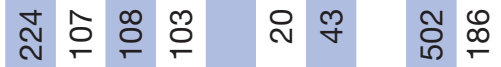
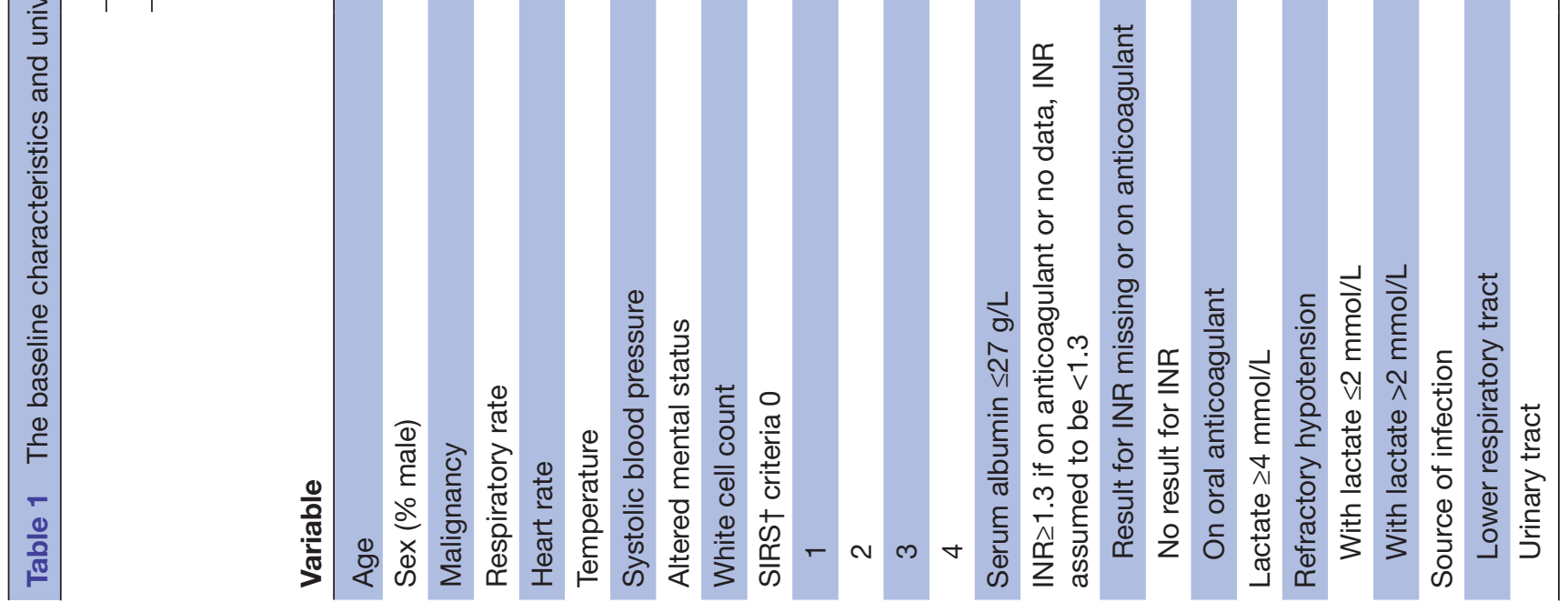


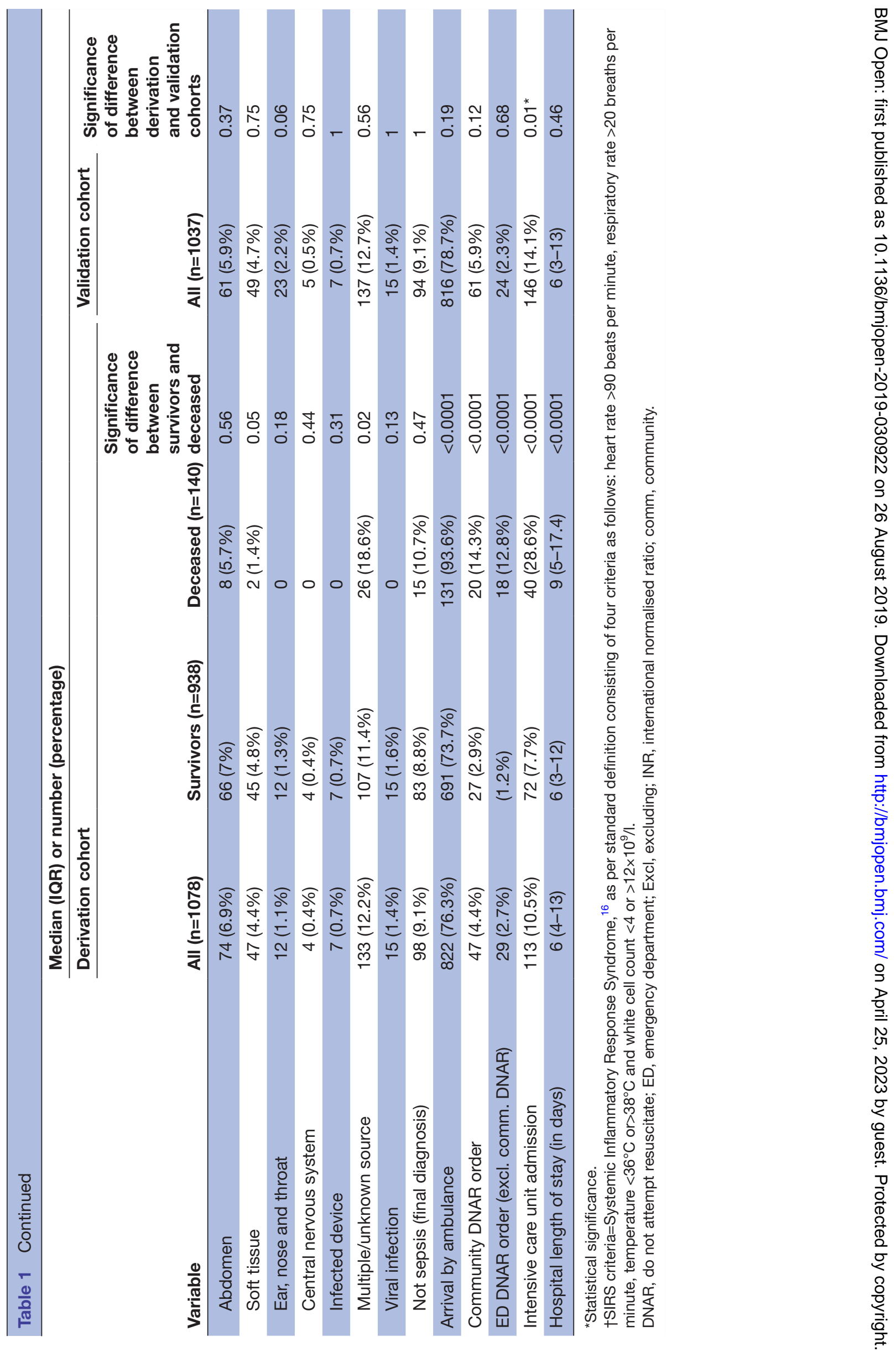




\begin{tabular}{|c|c|c|}
\hline Variable & Score & Guidance \\
\hline (1) Age $\geq 65 y$ & 1 & \\
\hline (2) Altered mental state & 1 & \multirow{3}{*}{ Use initial values or values measured at a given point in time } \\
\hline (3) Systolic blood pressure $\leq 100 \mathrm{~mm} \mathrm{Hg}$ & 1 & \\
\hline (4) Respiratory rate $\geq 22 / \mathrm{min}$ & 1 & \\
\hline $\begin{aligned} \text { (5) Lactate } & \leq 2 \mathrm{mmol} / \mathrm{l} \\
& 2.1-3.9 \mathrm{mmol} / \mathrm{l} \\
& \geq 4 \mathrm{mmol} / \mathrm{l}\end{aligned}$ & $\begin{array}{l}0 \\
1 \\
3\end{array}$ & Use initial or highest lactate \\
\hline (6) Albumin $\leq 27 \mathrm{~g} / \mathrm{l}$ & 1 & \\
\hline (7) INR $\geq 1.3$ & 1 & If on warfarin or a DOAC, score 0 \\
\hline $\begin{array}{l}\text { (8) No Refractory hypotension } \\
\text { Refractory hypotension and lactate } \leq 2 \mathrm{mmol} / \mathrm{l} \\
\text { Refractory hypotension and lactate }>2 \mathrm{mmol} / \mathrm{l}\end{array}$ & $\begin{array}{l}0 \\
2 \\
3\end{array}$ & $\begin{array}{l}\text { Refractory Hypotension }(\mathrm{RH})=\text { the requirement for } \\
\text { vasopressors to maintain MAP }>65 \mathrm{~mm} \mathrm{Hg} \text { after } 2 \mathrm{l} \text { fluid bolus. } \\
\text { Use a MAP }<62 \mathrm{mmHg} \text { after } 21 \text { fluid bolus as an indicator for the } \\
\text { need for vasopressors. } \\
\text { Use the lactate taken after the fluid bolus to stratify RH. }\end{array}$ \\
\hline REDS score & $-^{112}$ & \\
\hline
\end{tabular}

Figure 1 The Risk-stratification of Emergency Department suspected Sepsis (REDS) score. DOAC, directly acting oral anticoagulants; INR, international normalised ratio; MAP, mean arterial pressure; $\mathrm{RH}$, refractory hypotension.

was used to stratify RH. Mortality rates for each score 0-3 for each component score are shown in figure 2A.

UVA followed by MVLR confirmed all component scores to be independent predictors of mortality (table $2 \mathrm{~B}$ ). The
AUROC curve for the MVLR model was $0.79(95 \% \mathrm{CI} 0.76$ to 0.81 ); Hosmer-Lemeshow test confirmed good calibration, $\mathrm{p}=0.58$. The AUROC curve of the REDS score 0.78 $(95 \% \mathrm{CI} 0.75$ to 0.80$)$ was similar to that of the regression

\begin{tabular}{|c|c|c|c|c|}
\hline Score & 0 & 1 & 2 & 3 \\
\hline $\begin{array}{l}\text { qSOFA } \\
\text { (deaths) }\end{array}$ & $213(6)$ & $565(59)$ & $259(54)$ & 41 (21) \\
\hline SMISSED & $297(6)$ & $525(60)$ & $217(59)$ & $39(15)$ \\
\hline $\mathrm{RH}$ & 1015 (115) & NA & $20(5)$ & $43(20)$ \\
\hline Lactate & $676(69)$ & $299(38)$ & NA & $103(33)$ \\
\hline \multicolumn{5}{|c|}{$\begin{array}{l}\text { qSOFA=quick Sequential Organ Failure } \\
\text { Assessment score; sMISSED=simplified Mortality } \\
\text { in Severe Sep sis in the Emergency Department } \\
\text { score; RH- Refractory Hypotension; REDS= Risk- } \\
\text { stratification of Emergency Department } \\
\text { suspected Sepsis; NA=Not Applicable }\end{array}$} \\
\hline
\end{tabular}

B

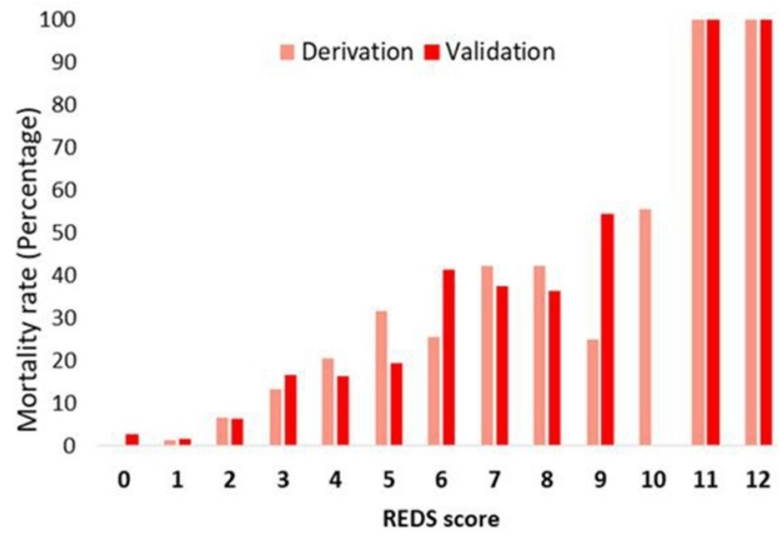

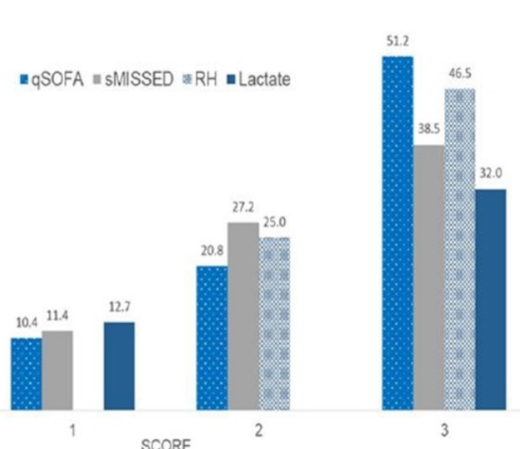

C

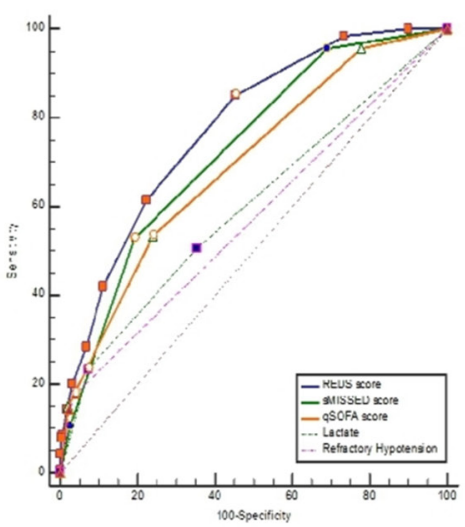
\begin{tabular}{|l|c|c|c|c|c|c|c|c|c|c|c|c|c|} 
Score & $\mathbf{0}$ & $\mathbf{1}$ & $\mathbf{2}$ & $\mathbf{3}$ & $\mathbf{4}$ & $\mathbf{5}$ & $\mathbf{6}$ & $\mathbf{7}$ & $\mathbf{8}$ & $\mathbf{9}$ & $\mathbf{1 0}$ & $\mathbf{1 1}$ & $\mathbf{1 2}$ \\
\hline Derivation & 92 & 158 & 283 & 249 & 132 & 60 & 47 & 19 & 19 & 4 & 9 & 5 & 1 \\
(Deaths) & $(0)$ & $(2)$ & $(19)$ & $(33)$ & $(27)$ & $(19)$ & $(12)$ & $(8)$ & $(8)$ & $(1)$ & $(5)$ & $(5)$ & $(1)$ \\
\hline & 72 & 126 & 280 & 254 & 135 & 67 & 46 & 24 & 11 & 11 & 6 & 4 & 1 \\
Validation & $(2)$ & $(2)$ & $(18)$ & $(42)$ & $(22)$ & $(13)$ & $(19)$ & $(9)$ & $(4)$ & $(6)$ & $(0)$ & $(4)$ & $(1)$ \\
\hline
\end{tabular}

Area under the Receiver Operator Characteristic curve (AUROC) were as follows: REDS score $0.78(95 \% \mathrm{CI} 0.75$ $0.80)$; sMISSED $0.73(0.7-0.75)$; qSOFA $0.70(0.67-0.72)$; Lactate 0.60 (0.57-0.63); Refractory Hypotension 0.57 $(0.54-0.6)$

Figure 2 Mortality rates associated with each score of (A) the component scores, (B) the REDS score and (C) the receiver operator characteristics of the REDS and component scores. 
Table 2A OR of high-risk and low-risk criteria of the component criteria

\begin{tabular}{|c|c|c|c|c|c|c|}
\hline \multirow[b]{2}{*}{ Score } & \multicolumn{2}{|c|}{ Low-risk scores 0-1 } & \multicolumn{2}{|c|}{ High-risk scores 2-3 } & \multirow[b]{2}{*}{ OR for mortality $(95 \% \mathrm{CI})$} & \multirow[b]{2}{*}{ Significance } \\
\hline & Number & Deaths & Number & Deaths & & \\
\hline qSOFA & 778 & 65 & 300 & 75 & 3.66 (2.54 to 5.26$)$ & $p<0.0001$ \\
\hline \multirow{2}{*}{ SMISSED } & \multicolumn{2}{|c|}{ Absence of $\mathrm{HL} / \mathrm{RH}$} & \multicolumn{2}{|c|}{ Presence of $\mathrm{HL} / \mathrm{RH}$} & OR for mortality (95\% Cl) & Significance \\
\hline & Number & Deaths & Number & Deaths & & \\
\hline Refractory hypotension & 1015 & 115 & 63 & 25 & 5.15 (3.00 to 8.84$)$ & $p<0.0001$ \\
\hline
\end{tabular}

$\mathrm{HL}$, high lactate $\geq 4 \mathrm{mmol} / \mathrm{L} ; \mathrm{RH}$, refractory hypotension; qSOFA, quick Sequential Organ Failure Assessment score; sMISSED, simplified Mortality in Severe Sepsis in the Emergency Department score.

Table 2B Univariate and multivariate logistic regression analysis of the component scores

\begin{tabular}{|c|c|c|c|c|c|c|}
\hline Score & $\begin{array}{l}\text { Alive } \\
\text { Median } \\
\text { (IQR) }\end{array}$ & $\begin{array}{l}\text { Dead } \\
\text { Median } \\
\text { (IQR) }\end{array}$ & $\begin{array}{l}\text { Significance } \\
\text { (univariate } \\
\text { analysis) }\end{array}$ & $\begin{array}{l}\text { Coefficient } \\
\text { of logistic } \\
\text { regression }\end{array}$ & $\begin{array}{l}\text { OR }(95 \% \mathrm{Cl}) \\
\text { for mortality on } \\
\text { logistic regression }\end{array}$ & Significance \\
\hline qSOFA & $1(1-1)$ & $2(1-2)$ & $<0.0001$ & 0.7178 & 2.05 (1.57-2.68) & $p<0.0001$ \\
\hline SMISSED & $1(0-1)$ & $2(1-2)$ & $<0.0001$ & 0.9358 & 2.55 (1.98-3.28) & $p<0.0001$ \\
\hline Lactate & $0(0-1)$ & $1(0-1)$ & $<0.0001$ & 0.2277 & 1.26 (1.04-1.52) & $p=0.02$ \\
\hline $\begin{array}{l}\text { Refractory } \\
\text { hypotension }\end{array}$ & $0(0-0)$ & $0(0-0)$ & $<0.0001$ & 0.2546 & $1.29(1.03-1.62)$ & $p=0.03$ \\
\hline
\end{tabular}

qSOFA, quick Sequential Organ Failure Assessment score; sMISSED, simplified Mortality in Severe Sepsis in the Emergency Department score.

model, negating the need to weight the component scores.

\section{The REDS score}

Range: $0-12$. Mortality rates associated with each score 0-12 for the REDS score are presented in figure 2B. The cut-off point was $\geq 3$. The REDS score had a greater AUROC and sensitivity for mortality in the high-risk category than its component scores (table 3). The ROC curves for the REDS score and the component scores are presented in figure $2 \mathrm{C}$.

Variants of the REDS score in the derivation cohort

Alternative qSOFA scores in the REDS score

There was no difference in AUROC curve or the cut-off points between REDS scores when the initial or final vital signs were used in the qSOFA score. However, when the highest RR and lowest SBP were used in the qSOFA score, the sensitivity for mortality for a qSOFA score of $\geq 2$ improved from $53 \%$ to $70 \%$. Incorporating this alternate qSOFA score, which included the most deranged values for RR and SBP over a period of time rather than the respective values at a given point in time, in the REDS score moved the cut-off point of the REDS score from 3 to 4 . This change in cut-off point in the REDS score significantly reduced the sensitivity for mortality in the highrisk category from $85 \%$ to $61.4 \%$. We therefore advocate calculating a qSOFA score using the variables measured at presentation (initial vital signs) or at a given point in time but not taking the worst variables over a period of time.
The highest lactate in the REDS score

Use of the highest lactate instead of the initial lactate did not significantly change the sensitivity for mortality of the high-risk category or the AUROC compared with the initial lactate. We therefore advocate the use of either the initial or highest lactate when calculating the REDS score.

$\mathrm{RH}$ as a homogeneous population in the REDS score

The overall mortality rate for RH was $39.7 \%$. This would warrant a score of 3 for all RH. Scoring 3 for all RH did not change the AUROC, the cut-off point or sensitivity for mortality of the REDS score. However, on MVLR it marginally reduces the significance of $\mathrm{RH}$ from 0.03 to 0.04 . We therefore advocate stratifying RH by the associated lactate as it is a more accurate reflection of the risk of death.

\section{Exclusion of patients with missing variables}

Exclusion of patients with missing variables improved the AUROC curve to 0.8 and improved the sensitivity for mortality in the high-risk group to $90 \%$, see table 3 . The mortality rate for a score of $0-2$ was $2.6 \%$.

\section{Validation study}

Of the 1060 patients between 5 October 2016 and 31 August 2017, ED notes were unavailable for 23 patients. Of the remaining 1037 patients, 142 died. Missing variables were lactate 42 , albumin 42 , INR or on oral anticoagulants 217. Of the 763 patients who had results for all variables, 96 died. 


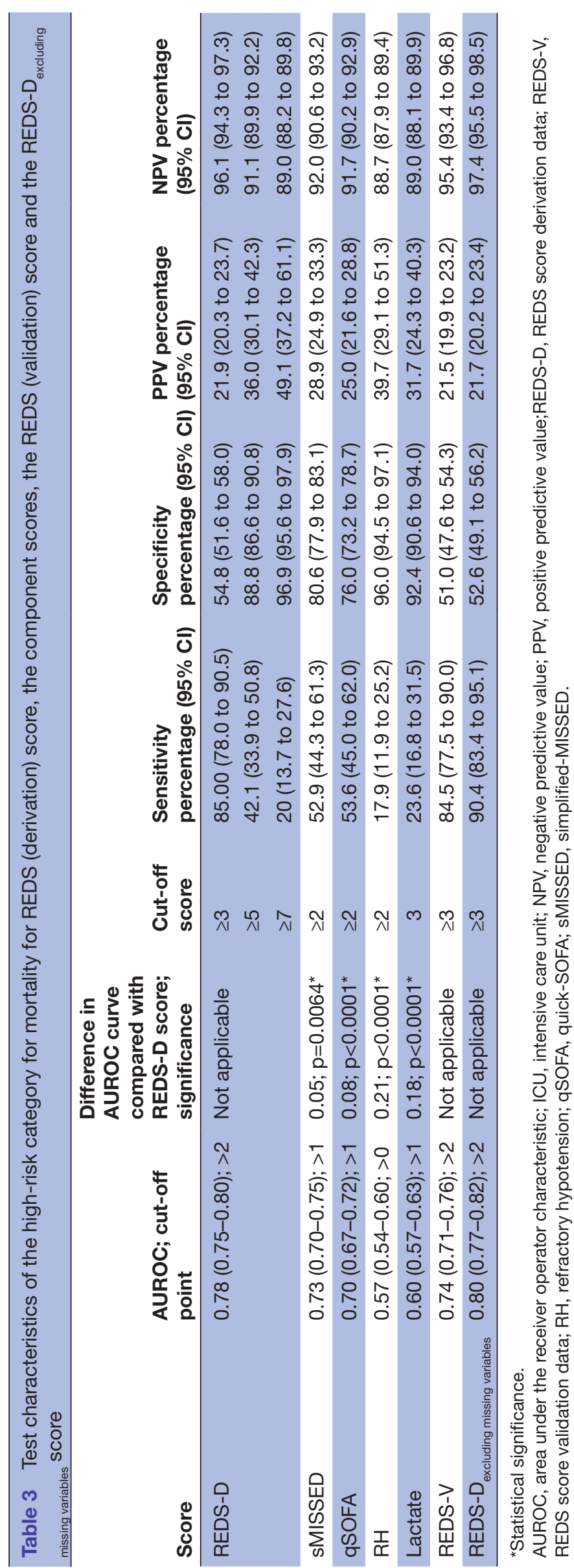


The AUROC curve of the regression model of the validation data was 0.74 (95\% CI 0.71 to 0.77 ) and the Hosmer-Lemeshow test showed good calibration, $\mathrm{p}=0.55$. See table 3 for the test-characteristics.

\section{Other findings}

Of the 2115 patients studied, 33 of the 44 patients who died with a REDS score of $0-2$ and 167 of the 202 who died without an admission to the ICU, had one or more of the following five comorbidities before admission: dementia, required significant help with daily living (nursing home (NH) residency or $\geq 3$ carer visits per day), community DNAR order, malignancy or were on long-term oxygen therapy. Three of the 73 patients with a score of 2 in one component score and a REDS score of 2, died. All three had community DNAR orders. Of the 164 patients with a score of 0 , there were two deaths and seven ICU admissions. The earliest death occurred on day 22.

Of the 259 patients admitted to the ICU during their hospital stay, 61 had scores of 0-2 while in the ED. The negative likelihood ratio for a REDS score of $>0$ for a combined endpoint of admission to the ICU or death was 0.18 (95\% CI 0.09 to 0.37 ).

Median HLOS for patients with a REDS scores of 3-12 was 8 days (IQR 4-16) compared with 5 days (IQR 3-9) for those with scores $0-2, \mathrm{p}<0.0001$.

\section{DISCUSSION}

In this study, we have derived and validated the REDS score which combines four component scores: the qSOFA score, the simplified MISSED score, lactate and RH. Each of the component scores ranged from 0 to 3 . The REDS score ranged from 0 to 12 . A REDS score of $\geq 3$ identified $85 \%$ (90\% if all variables were measured) of patients who go on to die in hospital and was associated with a mortality rate (positive predictive value) of over $21 \%$. A REDS score of 0-2 was associated with a mortality rate of $2.6 \%$ when all variables were measured. The specificity and positive predictive values for mortality in patients with REDS scores of $\geq 3, \geq 5$ and $\geq 7$ were $54.8 \%, 88.8 \%$ and $96.9 \%$, respectively. In addition, the REDS score had a better AUROC curve and sensitivity for mortality in the high-risk category than its component scores. The REDS score is easy to calculate in a busy ED as it uses widely available variables which are primarily dichotomous and it embraces the current definition of septic shock. ${ }^{22}$

It can be seen from table 1 that the baseline characteristics of the derivation and validation populations were similar except for a statistically significant difference in age and initial SBP. These two variables are key components of two of the component scores. Despite these differences, a REDS score of $\geq 3$ identified $85 \%$ of patients who died in each population.

While the population studied is large, there are several limitations to our study. It is a single-centre study and requires external validation. Patients not meeting the screening criteria and those who were discharged from the ED were not studied. Limiting the outcome to in-hospital mortality may have resulted in the underestimation of mortality as patients discharged before 28 days but died within 28 days would not have been recognised. ED treatments which may have influenced outcome and reason for ICU admission were not studied. Some ICU admissions may have occurred for logistical reasons when staffing skill-mix and bed availability in other areas of the hospital were limited. This may have contributed to the lower sensitivity for ICU admissions in the high-risk group. Furthermore, we did not exclude patients whose final diagnosis was not related to an infection. We took this pragmatic view as it would reflect reality. Over $23 \%$ of ICU admissions had REDS scores of $0-2$ while in the ED suggesting the REDS score may not pick-up patients who develop organ dysfunction. We also cannot advocate discharge from the ED based on a REDS score of 0 , as the negative likelihood ratio for a REDS score $>0$ is 0.18 for the composite endpoint of death or ICU admission. This statistic is not strong enough to support such action. Finally, we did not perform a sample size calculation to compare the AUROC curves. However, it is clear that the sensitivity for mortality of the REDS score above the cut-off point is greater than the sensitivities of the component scores above their respective cut-off points.

The AUROC curve of the sMISSED score for mortality was 0.73 , similar to previous reports. ${ }^{12} 13$ The AUROC for the qSOFA score was 0.7, similar to the AUROC of 0.71 found by Seymour et $a l^{5}$ for the community infections cohort. The sensitivity for mortality in our study for a qSOFA score of $\geq 2$ was $53.6 \%$ which increased to $70 \%$ when the highest RR and lowest SBP were used in the qSOFA score. This is similar to the $70 \%$ sensitivity for mortality found by other studies, ${ }^{67}$ where the worst reading for each variable was used. But this increased sensitivity for mortality was lost due to the increase in cut-off point of the REDS score from 3 to 4 . It is our view that measures for RR, SBP and mental state should be taken at a given point in time, rather than over a period of time and would reflect clinical use of the score. In our study, a lactate of $\geq 4 \mathrm{mmol}$ was associated with a $32 \%$ mortality rate which is similar to the $38 \%$ mortality rate found by Trzeciak et $a l,{ }^{21}$ who studied a population with a $19 \%$ mortality rate; a mortality rate higher than our study population. The mortality rates associated with the stratified RH of $25 \%$ and $46.5 \%$ for scores 2 and 3 , respectively, were similar to the mortality rates reported by Shankar-Hari et al. ${ }^{22}$

From a practical point, the calculation of the REDS score can only be completed after the initial treatment bundle (antibiotics and fluid) is complete and the results of the serum albumin and INR are available. Therefore, as the name suggests the REDS score is very much a risk stratification tool which aids disposition from the ED. The clinical use of the REDS score would be based on the specificities and mortality rates (positive predictive value) of the different scores: for those with a REDS score of $\geq 7$ (96.9\% specificity for mortality) ICU admission is 
mandatory. For those patients with REDS scores of $\geq 7$ in whom the admission to ICU is inappropriate, a DNAR order should be completed prior to leaving the ED. For those with a score of 5-6 (88.8\% specificity for mortality), ICU referral and transfer should be strongly considered. All patients with REDS scores of $\geq 3$ should have a treatment escalation plan to follow, should they deteriorate. Those with scores of 0-2 should follow the usual care pathway. Patients with RH should receive the recommended care. ${ }^{2}$

With regard to future developments, the REDS score requires external validation, in the first instance. Further study is required regarding the use of the REDS in patients who are already admitted and the use of the REDS score as a monitoring tool.

\section{CONCLUSION}

The REDS score is a new, simple and objective scoring system that uses widely available variables to risk-stratify ED patients suspected of having sepsis and is better than its component scores.

Acknowledgements We wish to thank Sally James, Orla Flaherty and Samara Hammond for helping with the data collection between mid-October and December 2016.

Contributors NS, LAB, SET and SC: gathering of data, analysis, interpretation and writing of the manuscript. ANH, MC and AR: analysis, interpretation and writing of the manuscript.

Funding The authors have not declared a specific grant for this research from any funding agency in the public, commercial or not-for-profit sectors.

Competing interests None declared.

Patient consent for publication Not required.

Ethics approval This study was deemed to be a service evaluation of routinely collected data and therefore ethics approval was not required.

Provenance and peer review Not commissioned; externally peer reviewed.

Data availability statement All data relevant to the study are included in the article or uploaded as supplementary information.

Open access This is an open access article distributed in accordance with the Creative Commons Attribution Non Commercial (CC BY-NC 4.0) license, which permits others to distribute, remix, adapt, build upon this work non-commercially, and license their derivative works on different terms, provided the original work is properly cited, appropriate credit is given, any changes made indicated, and the use is non-commercial. See: http://creativecommons.org/licenses/by-nc/4.0/.

\section{REFERENCES}

1. Singer M, Deutschman CS, Seymour CW, et al. The third International consensus definitions for sepsis and septic shock (Sepsis-3). JAMA 2016;315:801-10.
2. Rhodes A, Evans LE, Alhazzani W, et al. Surviving sepsis campaign: international guidelines for management of sepsis and septic shock: 2016. Crit Care Med 2017;45:486-552.

3. National Confidential Enquiry in to Patient Outcome and Death- 'Just say Sepsis', 2015. Available: www.ncepod.org.uk

4. Vincent J-L, Moreno R, Takala J, et al. The SOFA (sepsis-related organ failure assessment) score to describe organ dysfunction/ failure. Intensive Care Med 1996;22:707-10.

5. Seymour CW, Liu VX, Iwashyna TJ, et al. Assessment of clinical criteria for sepsis: for the third International consensus definitions for sepsis and septic shock (Sepsis-3). JAMA 2016;315:762-74.

6. Freund Y, Lemachatti N, Krastinova E, et al. Prognostic accuracy of Sepsis-3 criteria for in-hospital mortality among patients with suspected infection presenting to the emergency department. JAMA 2017;317:301-8.

7. Churpek MM, Snyder A, Han X, et al. Quick sepsis-related organ failure assessment, systemic inflammatory response syndrome, and early warning scores for detecting clinical deterioration in infected patients outside the intensive care unit. Am J Respir Crit Care Med 2017;195:906-11.

8. Song J-U, Sin CK, Park HK, et al. Performance of the quick sequential (sepsis-related) organ failure assessment score as a prognostic tool in infected patients outside the intensive care unit: a systematic review and meta-analysis. Critical Care 2018;22.

9. Fernando SM, Tran A, Taljaard M, et al. Prognostic accuracy of the quick sequential organ failure assessment for mortality in patients with suspected infection: a systematic review and meta-analysis. Ann Intern Med 2018;168:266-75.

10. Sivayoham N, Rhodes A, Cecconi M. The missed score, a new scoring system to predict mortality in severe sepsis in the emergency department: a derivation and validation study. Eur J Emerg Med 2014;21:30-6.

11. Ryoo SM, Ahn S, Kim WY, et al. External validation of the MISSED score to predict mortality in patients with severe sepsis and septic shock in the emergency department. Eur J Emerg Med 2015;22:327-30.

12. Sivayoham N, Holmes P, Cecconi M, et al. Internal emergency department validation of the simplified missed score. Eur J Emerg Med 2015;22:321-6.

13. McCormack D, Ruderman A, Menges W, et al. Usefulness of the mortality in severe sepsis in the emergency department score in an urban tertiary care hospital. Am J Emerg Med 2016;34:1117-20.

14. Rivers E, Nguyen B, Havstad S, et al. Early goal-directed therapy in the treatment of severe sepsis and septic shock. N Engl J Med 2001;345:1368-77.

15. Rowan KM, Angus DC, Bailey M, et al. Early, goal-directed therapy for septic shock - A patient-level meta-analysis. N Engl J Med 2017;376:2223-34

16. UK Sepsis Trust. Guidance for professionals. Available: https:// sepsistrust.org/professional-resources/clinical/

17. American College of chest Physicians/Society of critical care medicine consensus conference: definitions for sepsis and organ failure and guidelines for the use of innovative therapies in sepsis. Crit Care Med 1992;20:864-74

18. Hosmer DW, Lemeshow S. Applied logistic regression. Wiley, 2013: ISBN 978-0-470-58247-3.

19. DeLong ER, DeLong DM, Clarke-Pearson DL. Comparing the areas under two or more correlated receiver operating characteristic curves: a nonparametric approach. Biometrics 1988;44:837-45.

20. Stiell IG, Wells GA. Methodologic standards for the development of clinical decision rules in emergency medicine. Ann Emerg Med 1999;33:437-47.

21. Trzeciak S, Dellinger RP, Chansky ME, et al. Serum lactate as a predictor of mortality in patients with infection. Intensive Care Med 2007;33:970-7.

22. Shankar-Hari M, Phillips GS, Levy ML, et al. Developing a new definition and assessing new clinical criteria for septic shock: for the third International consensus definitions for sepsis and septic shock (Sepsis-3). JAMA 2016;315:775-87. 